\title{
Human amniotic mesenchymal stem cells improve ovarian function in natural aging through secreting hepatocyte growth factor and epidermal growth factor
}

Chenyue Ding ${ }^{1}$, Qinyan Zou', Fuxin Wang ${ }^{1}$, Huihua Wu', Rulei Chen², Jinghuan Lv², Mingfa Ling ${ }^{2}$, Jian Sun ${ }^{1}$, Wei Wang ${ }^{1}$, Hong Li ${ }^{1 *}$ and Boxian Huang ${ }^{1,2,3^{*}}$

\begin{abstract}
Background: Although many reports show that various kinds of stem cells have the ability to recover function in premature ovarian aging, few studies have looked at stem cell treatment of natural ovarian aging (NOA). We designed this experimental study to investigate whether human amniotic mesenchymal stem cells (hAMSCs) retain the ability to restore ovarian function, and how hAMSCs work in this process.

Methods: To build the NOA mouse model, the mice were fed for 12-14 months normally with young fertile female mice as the normal control group (3-5 months old). Hematoxylin and eosin staining permitted follicle counting and showed the ovarian tissue structure. An enzyme-linked immunosorbent assay was used to detect the serum levels of the sex hormones estradiol (E2), anti-mullerian hormone (AMH), and follicle-stimulating hormone (FSH). The proliferation rate and marker expression level of human ovarian granule cells (hGCs) (ki67, AMH, FSH receptor, FOXL2, and CYP19A1) were measured by flow cytometry (FACS). Cytokines (growth factors) were measured by a protein antibody array methodology. After hepatocyte growth factor (HGF) and epidermal growth factor (EGF) were co-cultured with hGCs, proliferation (ki67) and apoptosis (Annexin V) levels were analyzed by FACS. After HGF and EGF were injected into the ovaries of natural aging mice, the total follicle numbers and hormone levels were tested.

Results: After the hAMSCs were transplanted into the NOA mouse model, the hAMSCs exerted a therapeutic activity on mouse ovarian function by improving the follicle numbers over four stages. In addition, our results showed that hAMSCs significantly promoted the proliferation rate and marker expression level of ovarian granular cells that were from NOA patients. Meanwhile, we found that the secretion level of EGF and HGF from hAMSCs was higher than other growth factors. A growth factor combination (HGF with EGF) improved the proliferation rate and inhibited the apoptosis rate more powerfully after a co-culture with $\mathrm{hGCS}$, and total follicle numbers and hormone levels were elevated to a normal level after the growth factor combination was injected into the ovaries of the NOA mouse model.

Conclusions: These findings provide insight into the notion that hAMSCs play an integral role in resistance to NOA. Furthermore, our present study demonstrates that a growth factor combination derived from hAMSCs plays a central role in inhibiting ovarian aging. Therefore, we suggest that hAMSCs improve ovarian function in natural aging by secreting HGF and EGF.
\end{abstract}

Keywords: Natural ovarian aging, Human amniotic mesenchymal stem cells, HGF, EGF

\footnotetext{
*Correspondence: huangboxiannj@163.com (Boxian Huang); hongliszivf@163.com (Hong Li)

Chenyue Ding and Qinyan Zou are co-first authors.

${ }^{1}$ Center of Reproduction and Genetics, Affiliated Suzhou Hospital of Nanjing

Medical University, Suzhou Municipal Hospital, Suzhou 215002, China

Full list of author information is available at the end of the article
} 


\section{Background}

The ovary is a major regulator of female reproductive function. Its primary role is to provide a reserve of germ cells established prior to and shortly after birth which gradually decreases the quality and quantity of the oocytes that are contained in the follicles of the ovarian cortex during the period of natural ovarian aging (NOA) [1]. In humans, after the age of 38 years the progressive loss of ovarian follicles accelerates with age. Perimenopause is a midlife transition state experienced by women that results in reproductive senescence [2]. Following the loss of ovarian follicular activity, many perimenopausal symptoms occur, such as vaginal atrophy, osteoporosis, hot flushes, and depression [3].

Delayed childbearing is an important social change that has led to an increasing number of women desiring late menopause. Furthermore, women want to improve their quality of life by avoiding the trouble of perimenopausal symptoms and slowing down ovarian aging. During oocyte development, human granulosa cells (hGCs) play a key role in nurture and support [4]. GCs form the follicular microenvironment which facilitates oocyte development, supplies energy, disposes of waste, and participates in molecular signaling [5]. Research reveals that if the function of GCs becomes impaired with advanced age, oocyte growth and competence are compromised in parallel [6].

Currently, hormonal replacement therapy is used to treat common menopausal problems, but it increases the risk of cancer or recurrence in cancer survivors, forcing physicians to use alternative treatments [7]. Therefore, there is an urgent need to find an effective method to withstand NOA. Recent interest has grown in the therapeutic potential of stem cells, and multipotent stem cells can be developed from different sources such as adipose tissue, bone marrow, and amniotic fluid, all of which show huge therapeutic potential to restore ovarian function and rescue long-term fertility in chemotherapytreated female mice [8]. Human amniotic mesenchymal stem cells (hAMSCs) present several advantages which make them suitable for clinical therapy: they are an abundant tissue source, show little immunogenicity, are stably proliferative; thus, hAMSCs hold great promise for autologous cell repair and regeneration. AMSCs directly or indirectly (through a paracrine effect, releasing cytokines) participate in the regeneration process $[9,10]$. Previous studies show that hepatocyte growth factor (HGF) and epidermal growth factor (EGF) are secreted from multiple mesenchymal stem cells, such as hAMSCs, human amniotic fluid stem cells, and human menstrual blood stem cells [11].

Cytokine EGF is a member of the EGF family and is not only essential for cell proliferation, differentiation, growth, migration, and inhibiting apoptosis, but it also plays an important role in folliculogenesis [12, 13]. A previous study reported that EGF receptor (EGFR) is localized in the follicle cells in both mammals and fish $[14,15]$. EGF is reported to increase the proliferation rate of GCs in zebrafish follicles and enhance the cytoplasmic maturation of zebrafish oocytes, which are crucial for oocyte activation, fertilization, and early embryonic development [16]. HGF is a mesenchymederived multifunctional protein that plays a critical role in cell survival, proliferation, migration, and differentiation that acts through c-Met receptors [17, 18]. Previous studies suggest that HGF controls numerous key functions which collectively regulate the growth and differentiation of ovarian follicles [19].

Even so, much less is known about that whether hAMSCs retain the ability to resist NOA, and the relevant molecular mechanism of these effects is still not understood. To this end, in the present study, we identified whether hAMSCs maintain the ability to restore ovarian function and how hAMSCs work in this process.

\section{Methods \\ Preparation and culture of hAMSCs}

As previously described, hAMSC lines were established in our laboratory [20]. The cells were planted in a 10$\mathrm{cm}^{2}$ culture dish at a density of $1 \times 10^{7}$ in $10 \mathrm{ml}$ of regular growth medium [20] and were incubated at $37{ }^{\circ} \mathrm{C}$ and $5 \% \mathrm{CO}_{2}$. All the experiments were performed with passage 3-4 hAMSCs.

\section{Establishment of NOA mouse model}

Naturally aging female C57BL/6 mice (SPF class, 12-14 months old) and young female C57BL/6 mice with fertility (SPF class, 3-5 months old) were provided by the Nanjing Medical University with Institutional Animal Care and Use Committee approval in accordance with the institutional guidelines. They were bred at a temperature of $28 \pm 2{ }^{\circ} \mathrm{C}$ with a 12-h light/dark cycle. Vaginal smears of the mice were taken to determine the estrous cycle at 09:00 daily. Only aging mice with disorganized estrous cycles and young mice with normal estrous cycles were chosen. The aging mice were randomly distributed into the control group and cell treatment group ( $n=15$ per group). The young mice were set as the normal control group ( $n=15$ per group).

\section{Isolation of primary hGCs from NOA patients}

Young women (age $<40$ years) with tubal occlusion served as the control group $(n=31)$. The NOA patient selection followed a standard that the women were aged $>40$ years, had an antral follicle count $<5$ or antimullerian hormone $(\mathrm{AMH})<1.1 \mathrm{ng} / \mathrm{ml}$ and folliclestimulating hormone $(\mathrm{FSH}) \geq 10 \mathrm{mIU} / \mathrm{ml} \quad(n=26)$. Women with known normal karyotype, previous 
chemotherapy or radiotherapy, autoimmune diseases, or ovarian surgery were excluded. Primary hGCs were obtained following informed consent from the normal young women $(n=10)$ and NOA women $(n=15)$ after approval from the Suzhou Hospital Affiliated with the Nanjing Medical University Research Ethics Board. All the participants were treated with recombinant FSH (Puregon; Schering Plough, New Jersey, USA) and gonadotropin-releasing hormone $(\mathrm{GnRH})$ antagonist Ganirelix (Merck, Frosst, Montreal, Canada). Vaginal ultrasound examination was performed to monitor follicular development. Final follicular maturation was induced by administering 10,000 IU of human chorionic gonadotropin (Pregnyl, Merck). hGCs were purified using density centrifugation from follicular aspirates collected from women undergoing oocyte retrieval as previously described [20]. Primary hGCs were cultured in six-well plates in DMEM/F12 media (Thermo, USA) containing $1 \%$ penicillin/streptomycin, $10 \%$ fetal bovine serum (complete medium), $100 \mathrm{mg} / \mathrm{ml}$ streptomycin sulfate (Thermo, USA), and $1 \times$ GlutaMAX (Thermo, USA). The culture medium was changed every other day in all the experiments.

\section{HGF and EGF co-cultured with hGCs and injected into the} ovaries of NOA mice

Some of the abovementioned hGCs were divided into the HGF co-cultured group (treatment dose: $90 \mathrm{ng} / \mathrm{ml}$; Sigma), the EGF co-cultured group (treatment dose: $10 \mathrm{ng} / \mathrm{ml}$; R\&D system), the growth factor combination (HGF plus EGF) co-cultured group, and the phosphatebuffered saline (PBS) co-cultured control group. After 7 days, hGCs were used for study. The NOA mouse model was divided into the HGF-injected group (treatment dose: $0.9 \mu \mathrm{g} / \mathrm{ml}$; Sigma), the EGF-injected group (treatment dose: $0.1 \mu \mathrm{g} / \mathrm{ml} ; \mathrm{R} \& \mathrm{D}$ system), the growth factor combination-injected group, and a saline-injected control group. After 4 weeks, the mice in each group were killed to evaluate the follicle numbers by an hematoxylin and eosin (H\&E) assay.

\section{Assessment of ovarian function by a comparison of the ovarian follicle count}

After cell transplantation, the mice (cell transplanted and non-transplanted) were euthanized from 0 to 4 weeks; the ovaries on two sides were removed and fixed with $10 \%$ formalin, paraffin embedded, serially sectioned with a thickness of $5 \mu \mathrm{m}$, mounted in order on glass microscope slides, and stained with H\&E. Four stage follicles (primordial, primary, secondary, and antral follicles) were detected and classified. The ratio of the number of follicles from the ovary was calculated and compared between each group (per group, $n=15$ ). Three representative sections from each ovary were selected.
Only follicles containing an oocyte were counted to avoid counting any follicle twice. The experiments were repeated three times, and the results are presented as the foldchange $\pm \mathrm{SD} ; p<0.05$ determined a significant difference.

\section{Immunofluorescence staining}

The primary antibodies for anti-human-HGF (Abcam, USA), anti-human-transforming growth factor (TGF)B2 (Abcam, USA), anti-human-EGF (Abcam, USA), anti-human-Osteoprotegerin (Abcam, America), and anti-human-brain-derived neurotrophic factor (BDNF) (Abcam, USA) were selected to characterize ovarian tissues. For the staining procedure, the ovarian sections were fixed with $4 \%(\mathrm{w} / \mathrm{v})$ paraformaldehyde (PFA; Sigma, USA) at room temperature for $10 \mathrm{~min}$ and then washed three times for $5 \mathrm{~min}$ with PBS; they were then permeated with $0.1 \%$ Triton X-100 (Sigma, USA)/PBS on ice for 10 min and blocked with fresh $4 \%$ bovine serum albumin (BSA; Sigma, USA)/PBS at room temperature for $30 \mathrm{~min}$. The treated cells were washed with PBS three times for $5 \mathrm{~min}$ and were then incubated with primary antibodies overnight at $4{ }^{\circ} \mathrm{C}$. After rinsing with PBS for $5 \mathrm{~min}$, the cells were stained by $\mathrm{Cy} 2$ - or FITC-conjugated secondary antibodies (Jackson Immunoresearch, West Grove) in the dark at room temperature for $30 \mathrm{~min}$. The stained cells were mounted with 4',6-diamidino-2-phenylindole (DAPI; Vector Lab, USA) after washing with PBS for $5 \mathrm{~min}$ and were then photographed under a fluorescence microscope (Olympus, Japan).

\section{Antibody microarray analysis}

The cytokines were measured by a protein antibody array methodology (RayBio Human Cytokine Antibody Array, Human Growth Factor Array G1, RayBiotech, Inc., Norgross, GA) that contained antibodies to detect the protein expression level that was differentially expressed in the conditioned media (CM) from hAMSCs. One hundred micrograms of $\mathrm{CM}$ were used according to the instructions of the manufacturer.

\section{Flow cytometry (FACS) analysis}

The hGCs were digested separately by trypsin-EDTA for $3 \mathrm{~min}$ and were gently blown into single cells which were fixed and permeated by the Cytofix/Cytoper Fixation/ Permeabilization Solution Kit (BD, USA) following the instructions of the manufacturer. The hGCs were then stained with PE- or FITC-conjugated antibodies for antihuman-ki67 (Abcam, USA), anti-human-AMH (Thermo, USA), anti-human-FSH receptor (FSHR) (Thermo, USA), anti-human-Forkhead box L2 (FOXL2) (Thermo, USA), anti-human-CYP19A1 (Abgent, USA), and anti-humanAnnexin V (Abcam, USA) or their corresponding isotype control for $30 \mathrm{~min}$ at $4{ }^{\circ} \mathrm{C}$ as described above. These stained cells were analyzed on a fluorescence-activated 
cell sorter (FACS; Beckman, USA). The experiments were repeated three times, and the results are presented as the fold-change \pm SD; $p<0.05$ determined a significant difference.

\section{Enzyme-linked immunosorbent assay (ELISA) analysis}

Plasma from the NOA mouse model was harvested after hAMSC transplantation or growth factor (EGF, or HGF, or combination) injection to evaluate the expression level of estradiol (E2), AMH, or FSH using an ELISA kit (Mybiosource, USA) according to the manufacturer's instructions. Briefly, $50 \mu \mathrm{l}$ of the serum sample was added per well. The test plate was wrapped with a membrane and incubated for $30 \mathrm{~min}$ at $37^{\circ} \mathrm{C}$. Thereafter, the wells on the plate were dried and washed with Wash Buffer five times (10 s per wash). Then, $50 \mu \mathrm{l}$ of the HRPconjugated reagent was added into each sample well and incubated for $60 \mathrm{~min}$ at $37^{\circ} \mathrm{C}$. The samples were washed with Wash Buffer five times (10 s per wash). Subsequently, $50 \mu \mathrm{l}$ of substrate A Solution followed by $50 \mu \mathrm{l}$ of substrate B Solution were added and incubated for $15 \mathrm{~min}$ at $37^{\circ} \mathrm{C}$. Then, $50 \mu \mathrm{l}$ of Stop Solution was added into each control and the sample well. Finally, the light absorbance was measured and recorded by a spectrophotometer (Varian Company, Australia).

\section{Statistical analysis}

All the results are shown as means \pm SD. Statistically significant differences were determined by a one-way analysis of variance (ANOVA) with SPSS 17.0 software, and $p<0.05$ was regarded as being statistically significant.

\section{Results \\ hAMSCs restored ovarian function in the NOA mice model}

First, the hAMSC lines were established successfully as described in a previous paper [20]. After the hAMSCs were injected into the ovaries of the NOA mice, the H\&E stained ovarian tissues showed that the hAMSCs restored the follicle numbers to $21 \%$ for primordial follicles, $42 \%$ for primary follicles, $58 \%$ for secondary follicles, and $46 \%$ for antral follicles at week 1 compared with the levels in the control group (Fig. 1). In contrast, after the hAMSCs were injected for 4 weeks, our results showed that the hAMSCs recovered the follicle numbers to $88 \%$ for primordial follicles, $89 \%$ for primary follicles, $86 \%$ for secondary follicles, and $81 \%$ for antral follicles compared with the levels in the control group which was a significant improvement $(p<0.01)$ compared with the NOA group (Fig. 1).

The hormone levels of the plasma in each group was tested after hAMSC transplantation. In the treatment group, an ELISA assay demonstrated that the level of E2 (72\%) and AMH (77\%) was slightly rescued compared to the aging group (50\% E2 and 52\% $\mathrm{AMH}$ ) and to that of the control group at week 1 (Fig. 2a, c). However, at week 4, the hAMSCs rescued the level of E2 (95\%) and $\mathrm{AMH}(92 \%)$ to the normal levels of the control group
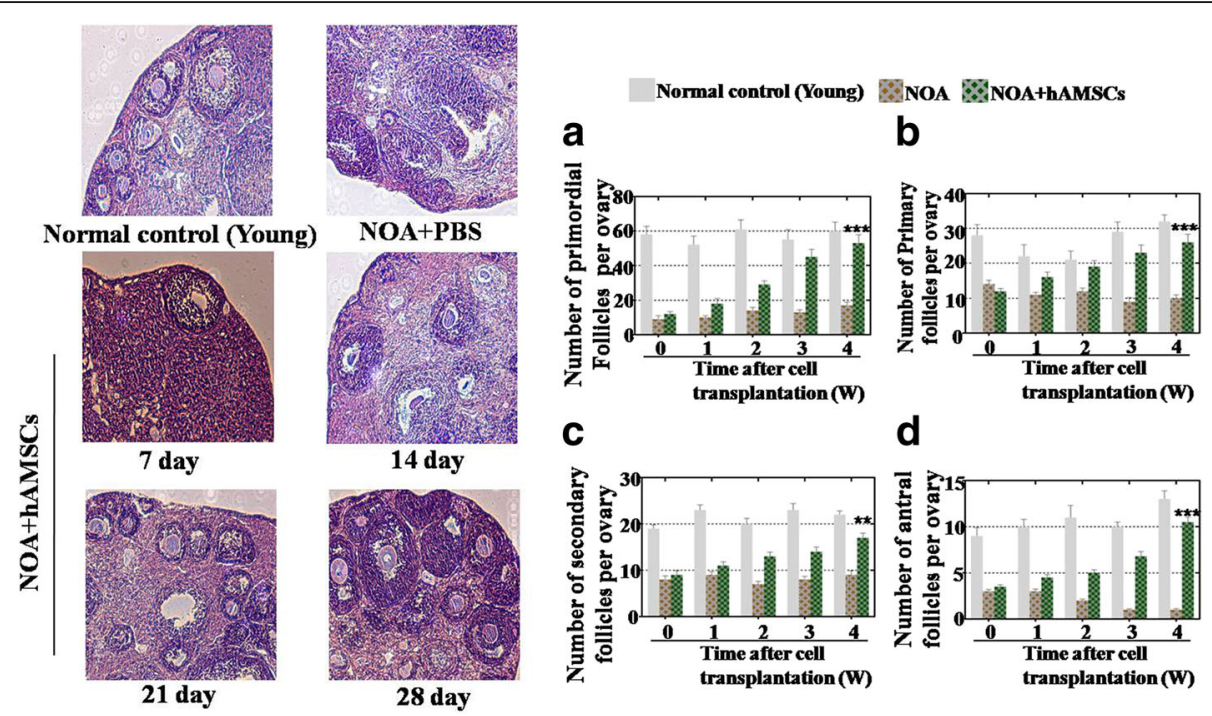

Fig. 1 Human amniotic mesenchymal stem cells (hAMSCs) improve the follicle numbers in the ovaries of a natural ovarian aging (NOA) mouse model at four stages. a The number of primordial follicles was counted over 4 weeks after hAMSC transplantation. $\mathbf{b}$ The number of primary follicles was counted over 4 weeks after hAMSC transplantation. c The number of secondary follicles was counted over 4 weeks after hAMSC transplantation. $\mathbf{d}$ The number of antral follicles was counted over 4 weeks after hAMSC transplantation. All the experiments were repeated three times; the error bars indicate SD. ${ }^{* *} p<0.01,{ }^{* *} p<0.001$, versus the NOA group. PBS phosphate-buffered saline 

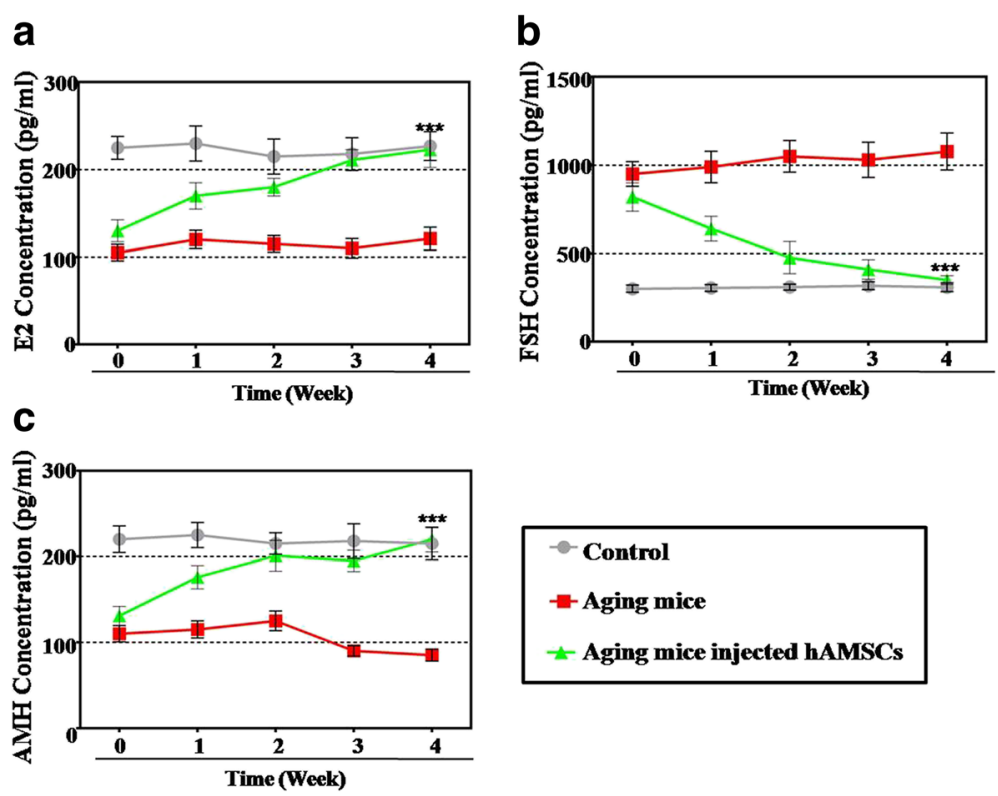

Fig. 2 Human amniotic mesenchymal stem cells (hAMSCs) improve the hormone levels in the ovaries of an NOA mouse model. a The level of estradiol (E2) was measured by ELISA over 4 weeks after hAMSC transplantation. b The hormonal level of follicle-stimulating hormone (FSH) was measured by ELISA over 4 weeks after hAMSC transplantation. c The hormonal level of anti-mullerian hormone (AMH) was measured by ELISA over 4 weeks after hAMSC transplantation. All the experiments were repeated three times; the error bars indicate SD. ${ }^{* * *} p<0.001$, versus the aging mice group

which was a significant improvement $(p<0.001)$ compared with the NOA group (Fig. 2a, c). In addition, the level of FSH decreased to $209 \%$ of that in the treatment group compared to $325 \%$ in the aging mice group at week 1 . In contrast, after hAMSC treatment for 4 weeks, the level of FSH was rescued to the normal control group level (109\%) which was a significant improvement $(p<0.001)$ compared with the NOA group (Fig. 2b).

Overall, the hAMSCs exhibited a powerful ability to restore ovarian function in an NOA mouse model.

\section{hAMSCs increased the proliferation rate and marker expression of the hGCs}

To investigate the therapeutic effects of hAMSCs in NOA patients in the clinic, we collected hGCs from a young group $(n=19)$ and an NOA patient group $(n=15)$ in our reproductive center to examine the effects of cell proliferation after co-culturing with hAMSCs (Fig. 3a). hGC markers (AMH, FSHR, FOXL2, and CYP19A1) and a ki67 antibody (cell proliferation marker) were used to estimate the effects from hAMSCs by a FACS analysis method. Our results showed that hAMSCs increased the ki67 ${ }^{+} \mathrm{AMH}^{+}$cell number in the NOA group to a higher level $(81 \%, p<0.001)$ than the PBS administration group (33\%) compared with the young group (Fig. 3b). The FACS assay results demonstrated that hAMSCs increased the $k i 67^{+} \mathrm{FSHR}^{+}$cell number to $86 \%(p<0.001)$ in the
NOA group, higher than the $32 \%$ in the PBS administration group compared with that of the young group (Fig. 3c). Our results further revealed that hAMSCs increased the ki67 $7^{+} \mathrm{FOLX2}{ }^{+}$cell number in the NOA group to a greater degree $(97 \%, p<0.001)$ than the $48 \%$ seen in the PBS administration group compared with that of the young group (Fig. 3d). In addition, the FACS assay results showed that the hAMSCs raised the ki6 $7^{+} \mathrm{CYP} 19 \mathrm{~A} 1^{+}$cell number to $83 \%(p<0.001)$ in the NOA, higher than the $32 \%$ in the PBS co-cultured group compared with that of the young group (Fig. 3e).

Overall, the hAMSCs showed powerful recovery effects for natural senescent hGCs.

\section{hAMSC-derived HGF and EGF levels were higher than other growth factors}

To investigate the mechanism of the restoring effect of transplanting hAMSCs into NOA mice, the supernatant was collected from three hAMSC lines derived from three different donors (two male (hAMSCs-04, -05), one female (hAMSCs-01)) and the control group (293 T cell line), and then a cytokine antibody array (growth factors $=52$ ) was utilized to evaluate the diversity. Our analytical data elucidated that the large number of growth factors in the hAMSCs exceeded the control group (Additional file 1: Figure S1A). There were 23 growth factors from the hAMSCs that were secreted at a 


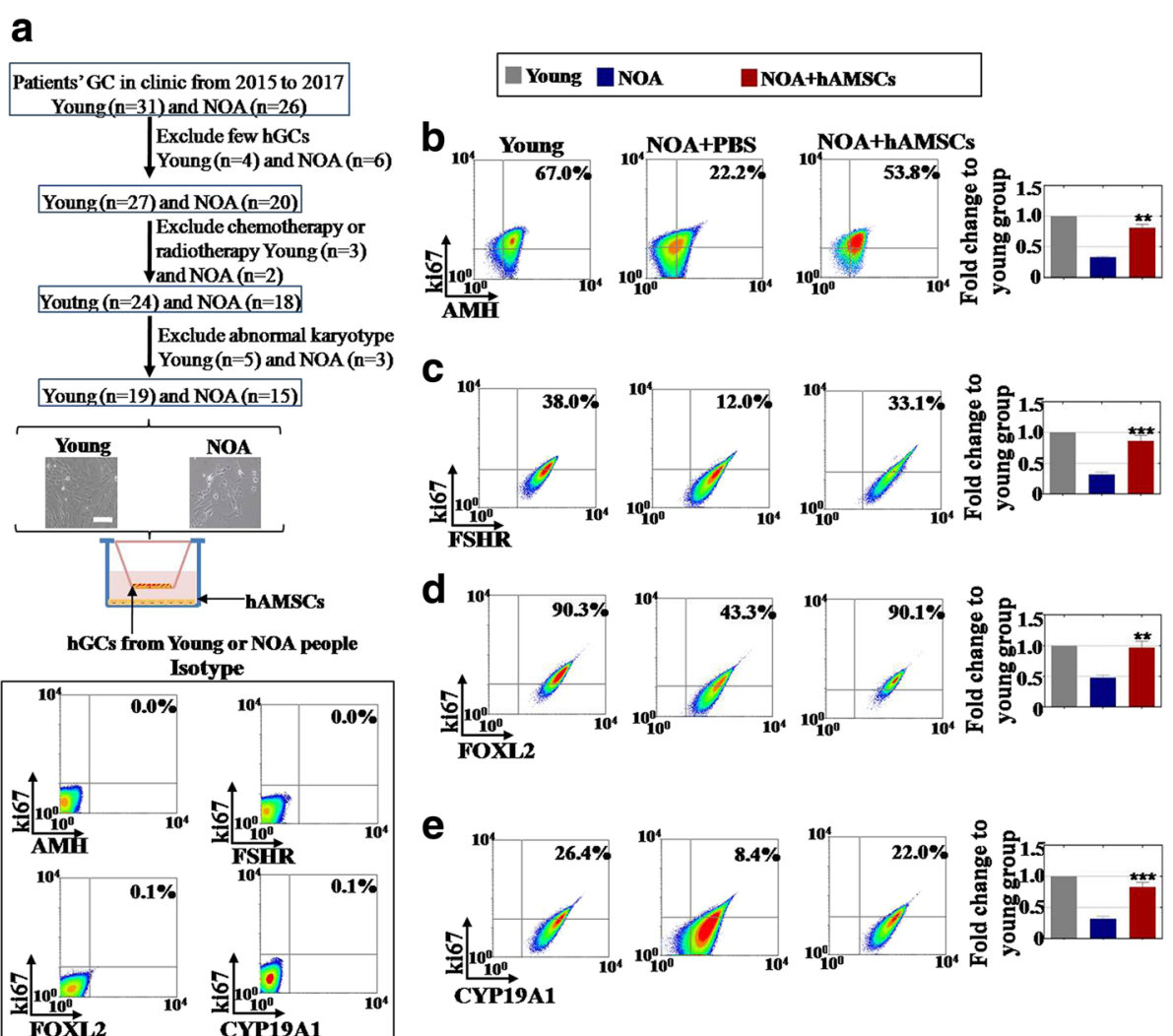

Fig. 3 Human amniotic mesenchymal stem cells (hAMSCs) improve the proliferation rate of human granulosa cells (hGCs) and upregulate the expression of hGC markers. a A schematic overview of the hGC filter procedures. $\mathbf{b}$ The number of ki67 ${ }^{+} \mathrm{AMH}^{+} \mathrm{hGCS}$ was evaluated after a co-culture with hAMSCs. c The expression level of the ki67 ${ }^{+} \mathrm{FSHR}^{+} \mathrm{hGCs}$ was tested after a co-culture with hAMSCs. $\mathbf{d}$ The expression level of the ki67 ${ }^{+} \mathrm{FOXL2}{ }^{+}$hGCs was tested after a co-culture with hAMSCs. e The number of ki67 ${ }^{+} \mathrm{CYP19A} 1^{+}$hGCs was evaluated after a co-culture with hAMSCs. The experiments were carried out after 7 days of co-culture, $n=3$; error bars indicate SD. ${ }^{* *} p<0.01$, ${ }^{* * *} p<0.001$, versus the NOA group. AMH anti-mullerian hormone, FOXL2 forkhead box L2, FSHR follicle-stimulating hormone receptor, NOA natural ovarian aging, PBS phosphate-buffered saline

significantly higher level than in the control group $(p<$ 0.05) (Additional file 1: Figure S1B). In accordance with standard criteria of fold-change greater than or equal to 8 and statistical significance $(p<0.01)$, five growth factors were further selected: BDNF, EGF, Osteoprotegerin, HGF, and TGF- $\beta 2$ (Additional file 1: Figure S1B). The secretion level of EGF and HGF was obviously higher than the other growth factors (Fig. 4). After the hAMSCs were transplanted into the NOA mice for 4 weeks, an immunofluorescence assay revealed that EGF and HGF were expressed in ovaries of the NOA mice, but not BDNF, Osteoprotegerin, or TGF-ß2 (Fig. 5).

Taken together, these results suggest that EGF and HGF might play a vital role in improving ovary function of natural aging mice.

HGF and EGF combined improved the proliferation rate and inhibited the apoptosis rate in hGCs

To examine the effects of HGF and EGF on cell proliferation and apoptosis, hGCs were co-cultured with
HGF and EGF for 7 days (Fig. 6a). A FACS assay was employed to quantitatively assess cell viability. Our results showed that the proliferation rate of the hGCs was raised to $21 \%$ in the HGF group and 19\% in the EGF group, which was higher than the untreated group (9\%), compared with that of the young normal control mice group at 70\% (Fig. 6b). The FACS assay also showed that the growth factor combination group (HGF plus EGF) increased the proliferation rate of the hGCs to a higher degree (reaching 67\%), significantly higher than the HGF-alone and EGF-alone groups $(p<0.001$, Fig. 6b). Similarly, the results from the apoptosis assay indicated that a single growth factor decreased the rate of apoptosis to $34 \%$ in the HGF group and $38 \%$ in the EGF group, which was lower than the untreated group (61\%), compared with of the young mice group at $8 \%$ (Fig. 6c). The combined group of growth factors inhibited the rate of apoptosis more strongly at $14 \%(p<$ 0.01 ), significantly different from the HGF and EGF groups (Fig. 6c). 


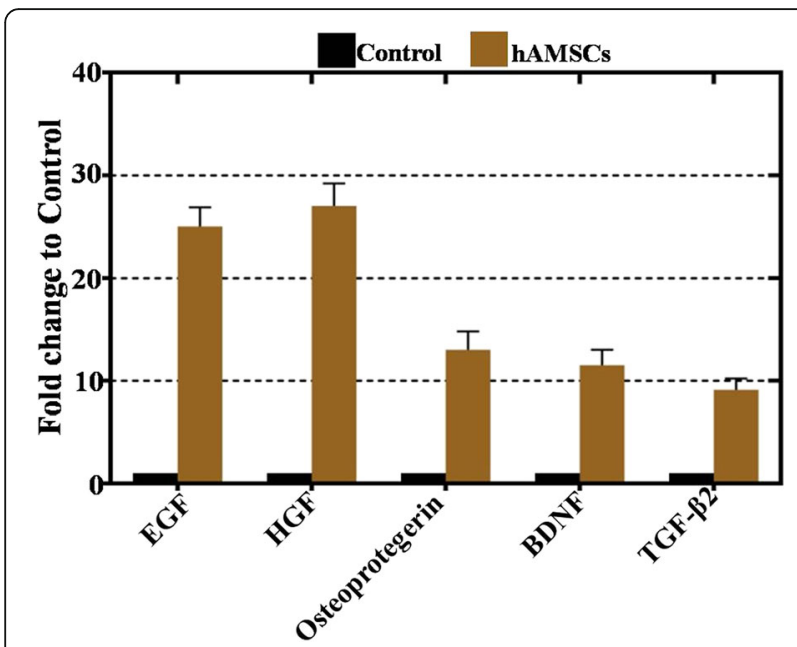

Fig. 4 Epidermal growth factor (EGF) and hepatocyte growth factor (HGF) derived from the human amniotic mesenchymal stem cells (hAMSCs) were higher than other growth factors. HGF and EGF derived from the hAMSCs exhibited a higher level of secretion than other growth factors. All the experiments were repeated three times; error bars indicate SD. BDNF brain-derived neurotrophic factor, TGF transforming growth factor

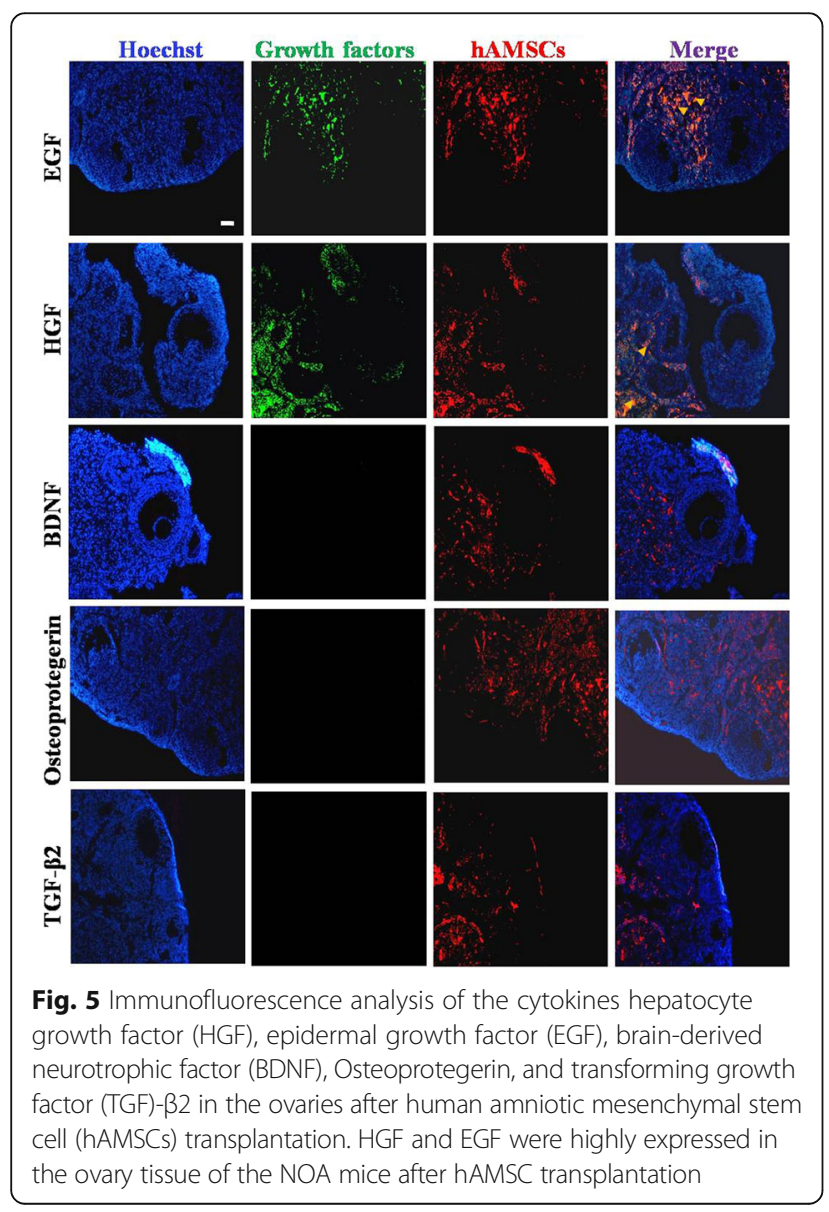

In summary, a combination of two growth factors increased the proliferation rate and inhibited the apoptosis rate of hGCs more effectively than using them singly.

\section{The HGF and EGF combination group increased the} follicle number and improved the hormone level in NOA mice

The follicle numbers were counted after the HGF and EGF injection into the ovaries of a natural aging mouse model. Our result showed that using the growth factors singly increased the number of follicles to $35 \%$ in the HGF group and $41 \%$ in the EGF group, which was a little more than the natural aging group (19\%) compared with that in the young mice group (Fig. 7a). After the growth factor combination was injected into the ovaries of the NOA mice, we found that the number of follicles increased to $85 \%$ of that in the young control mice, which was significantly different to the values in the HGF and EGF single groups $(p<0.01)$ (Fig. 7a). Furthermore, the hormone levels in the plasma in each group were also determined after HGF and EGF injections. The ELISA assay results revealed that the level of E2 and AMH increased moderately in the HGF group (reaching 52\% and $55 \%$ of the value in the control group), the EGF group (reaching $49 \%$ and $48 \%$ of the value in the control group), and the growth factor combination group (reaching 59\% and 68\% of the value in the control group), which was moderately higher than the untreated group (reaching $40 \%$ and $42 \%$ of the value in the control group) after injections for 1 week (Fig. 7b, d). However, after HGF and EGF injection for 4 weeks, the level of E2 and $\mathrm{AMH}$ increased to $68 \%$ and $72 \%$ of the value in the EGF group, and $69 \%$ and $73 \%$ of the value in the HGF group. In contrast, the growth factor combination rescued the levels of E2 and AMH to normal (98\% and 99\% of the value in the control group), with no improvement seen in the young mice group. The combination injection was significantly higher than the single growth factor injections $(p<0.01$ for E2; $p<0.05$ for $\mathrm{AMH})$ (Fig. 7b, d). This finding was also seen for FSH. Our results showed that the level of FSH increased to $273 \%$ in the HGF group, $298 \%$ in the EGF group and $284 \%$ in the growth factor combination group, results which were a little lower than that of the aging group (369\%), compared with that of the young mice group after an injection for 1 week (Fig. 7c). However, after HGF and EGF injection for 4 weeks, a single growth factor inhibited the levels of FSH to just $237 \%$ in the HGF group and $248 \%$ in the EGF group compared to the young mice group, whereas the growth factor combination rescued the level of FSH to normal levels (98\% of the control group) compared to the young mice group at week 4 , significantly lower than either single growth factor injection group $(p<$ 0.01) (Fig. 7c). 

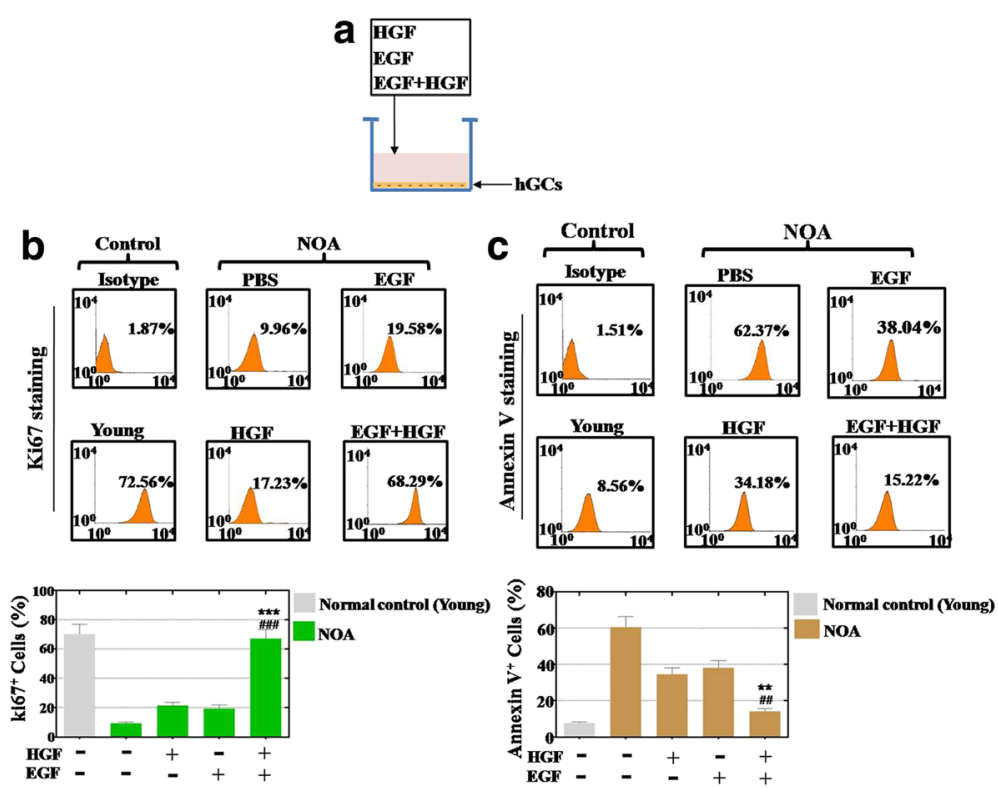

Fig. 6 A combination of epidermal growth factor (EGF) and hepatocyte growth factor (HGF) improves proliferation and inhibits the apoptosis level to a greater degree than using them alone after a co-culture with human granulosa cells ( $h G C s$ ). a A schematic diagram of the EGF and HGF co-culture with hGCS. b A combination of EGF and HGF improved proliferation in the hGCs more significantly than using them singly. c A combination of EGF and HGF inhibited apoptosis in the hGCs more effectively than using them singly. All the experiments were repeated three times; error bars indicate SD. ${ }^{* *} p<0.01,{ }^{* * *} p<0.001$, versus the HGF group; ${ }^{\# \#} p<0.01,{ }^{\# \# \#} p<0.001$, versus the EGF group. NOA natural ovarian aging, PBS phosphate-buffered saline

Taken together, these results suggest that the growth factor combination group played a vital role in improving ovary function in a natural aging mouse model.

\section{Discussion}

Various types of stem cells have been used to restore premature ovarian failure, including human amniotic epithelial cells, bone marrow mesenchymal stem cells, human amniotic fluid stem cells, and human menstrual blood stem cells [11]. However, it is still not understood whether hAMSCs retard NOA and how hAMSCs work. In this study, after hAMSC transplantation, the follicle numbers at four stages were nearly recovered to normal levels in the NOA mice. We analyzed the serum index of $\mathrm{E} 2, \mathrm{AMH}$, and $\mathrm{FSH}$, and all these were restored to normal levels (Figs. 1 and 2). In addition, to bridge the bench-to-bedside gap, the preclinical efficacy of hAMSCs in delaying NOA were evaluated, and hGCs that derived from NOA patients were co-cultured with hAMSCs. Our findings indicate that hAMSCs increased the proliferation rate and marker expression of hGCs to natural levels (Fig. 3). Our research, therefore, firstly confirmed that hAMSCs have ability to withstand NOA.

Although data from several studies suggest that hAMSCs plays a pivotal role in wound healing, antineural aging, and angiogenesis [9, 10, 21], few studies reveal the mechanisms by which hAMSCs achieve these results. Specifically, little is known about the mechanism of how hAMSCs resist NOA. According to a previous reports, AMSCs withstand premature aging and reveal an antioxidant ability via secretory cytokines [22-24]. In our results, antibody microarray results revealed that growth factor HGF and EGF secretions from hAMSCs were expressed at a high level (Fig. 4). Meanwhile, after hAMSC transplantation, HGF and EGF were highly expressed in the ovaries of natural aging mice (Fig. 5). Several lines of evidence support that higher levels of HGF and EGF are secreted by mesenchymal stem cells $[22,25]$.

Even so, there is no reliable evidence whether HGF and EGF function to withstand NOA. To address this issue further, a growth factor combination group was used. After a growth factor combination was added to the hGCs, our FACS results revealed that HGF plus EGF improved the proliferation rate and reduced the apoptosis rate of the hGCs more robustly than using either factor alone (Fig. 6). Furthermore, an ovarian function study also showed similar results. When HGF and EGF were injected into the ovaries of natural aging mice together, the total follicle number and the hormone levels were elevated more notably than by using a single growth factor (Fig. 7). Moreover, earlier research also reveals that EGF-like growth factors play a critical role in peri-ovulatory events in humans [14]. HGF is a known 


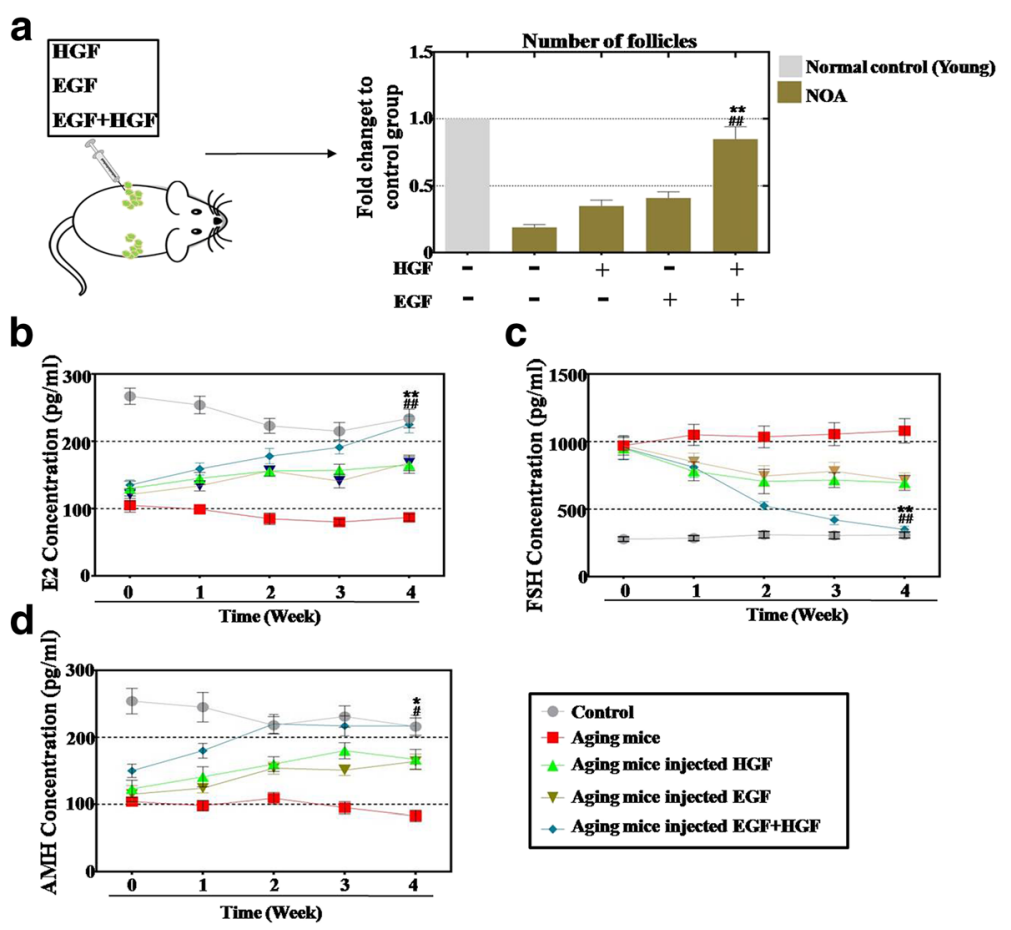

Fig. 7 A combination of epidermal growth factor (EGF) and hepatocyte growth factor (HGF) increases the follicle numbers and elevates the hormone levels to a greater extent than using them alone after an injection into a natural ovarian aging (NOA) mouse model. a A combination of EGF and HGF increased the follicle numbers to a higher level than using them singly. $\mathbf{b}$ A combination of EGF and HGF increased the level of estradiol (E2) to a higher level than using them singly. c A combination of EGF and HGF increased the level of follicle-stimulating hormone (FSH) to a higher level than using them singly. $\mathbf{d}$ A combination of EGF and HGF increased the level of anti-mullerian hormone (AMH) to a higher level than using them singly. All the experiments were repeated three times; error bars indicate SD. ${ }^{*} p<0.05,{ }^{* *} p<0.01$, versus the HGF group; ${ }^{\#} p<$ $0.05, " \# p<0.01$, versus the EGF group

crucial regulator of anti-apoptosis in preantral follicles [26]. These current findings are consistent with results from a previous study showing that HGF and EGF promote cell proliferation and delay oocyte aging during culture in vitro $[27,28]$.

\section{Conclusions}

We investigated the interaction between hAMSCs and NOA for the first time. The results of the present study provide insight into the notion that hAMSCs delay NOA by secreting HGF and EGF. Furthermore, our present study revealed that a growth factor combination derived from hAMSCs plays a central role in inhibiting ovarian aging. Therefore, we suggest that hAMSCs improve the ovarian function of natural aging by secreting HGF and EGF. This discovery has important implications for understanding the molecular mechanisms by which growth factors promote the ovarian function in natural aging. Moreover, this discovery suggests that HGF and EGF may serve as a novel, safe, and efficacious therapeutic schedule to resist NOA and improve female reproductive health.

\section{Additional file}

Additional file 1: Figure S1. An antibody microarray to test the level of the growth factors derived from the hAMSCs. (A) Antibody microarray analysis of the growth factor secretion from the hAMSCs and the control group. (B) Five growth factors were selected in accordance with the standard criteria that the fold-change was greater than or equal to 8 and was statistically significant $(p<0.01)$. (TIF $26685 \mathrm{~kb}$ )

\section{Abbreviations}

AMH: Anti-mullerian hormone; BDNF: Brain-derived neurotrophic factor; CM: Conditioned media; CYP19A1: Cytochrome P450 family 19 subfamily A member 1; E2: Estradiol; EGF: Epidermal growth factor; EGFR: Epidermal growth factor receptor; ELISA: Enzyme-linked immunosorbent assay; FACS: Fluorescence-activated cell sorter; FOXL2: Forkhead box L2; FSH: Follicle-stimulating hormone; FSHR: Follicle-stimulating hormone receptor; hAMSC: Human amniotic mesenchymal stem cell;

H\&E: Hematoxylin and eosin; hGC: Human granulosa cell; HGF: Hepatocyte growth factor; NOA: Natural ovarian aging; PBS: Phosphate-buffered saline; TGF: Transforming growth factor

\section{Acknowledgements}

Not applicable.

\section{Funding}

This work was supported by grants from Suzhou Science and Technology for People's Livelihood (SYS201771), Suzhou Science and Technology for People's Livelihood, Suzhou Key Medicine Center (SZZX201505), Suzhou Health Bureau Fund (Iczx201411), Maternal and Health Care Project of Jiangsu Province- 
Assisted Reproduction Medicine Center (FKX201224), Jiangsu Provincial Medical innovation team (CXTDB2017013), project funded by China Postdoctoral Science Foundation (2017 M611871), and Suzhou Introduce Expert Team of Clinical Medicine (SZYJTD201708).

\section{Availability of data and materials}

All the data generated and/or analyzed during this study are included in this published article.

\section{Authors' contributions}

CD performed the cellular and molecular assays in vivo and in vitro. QZ participated in the statistical analysis and revised the manuscript. FW contributed to hGC collection and purification. HW contributed to hGC purification culture. RC carried out the partial immunoassays. JL carried out the partial H\&E assays. ML participated in the mice feeding. JS participated in the cell culture. WW performed experiments and analyzed data. $\mathrm{HL}$ planned the experiments and drafted part of the manuscript. $\mathrm{BH}$ planned the experiments and wrote the manuscript. All the authors read and approved the final manuscript.

\section{Ethics approval and consent to participate}

The use of human ovarian granular cells and human peripheral blood mononuclear cells were in accordance with the relevant guidelines and regulations, and the experimental protocols were approved by the Medical Ethics Committee of the Suzhou Hospital Affiliated to Nanjing Medical University (NJMU-2015-014). All the patients provided written informed consent prior to participation in this study.

Our investigation using experimental animals was conducted on the basis of the Nanjing Medical University Animal Center's specific guidelines and standards.

\section{Consent for publication}

Not applicable.

\section{Competing interests}

The authors declare that they have no competing interests.

\section{Publisher's Note}

Springer Nature remains neutral with regard to jurisdictional claims in published maps and institutional affiliations.

\section{Author details}

${ }^{1}$ Center of Reproduction and Genetics, Affiliated Suzhou Hospital of Nanjing Medical University, Suzhou Municipal Hospital, Suzhou 215002, China. ${ }^{2}$ Central Laboratory, Affiliated Suzhou Hospital of Nanjing Medical University, Suzhou Municipal Hospital, Suzhou 215002, China. ${ }^{3}$ State Key Laboratory of Reproductive Medicine, Nanjing Medical University, Nanjing 210029, China.

Received: 25 July 2017 Revised: 12 December 2017 Accepted: 16 January 2018 Published online: 09 March 2018

\section{References}

1. Nelson SM, Telfer EE, Anderson RA. The ageing ovary and uterus: new biological insights. Hum Reprod Update. 2013;19:67-83.

2. Chung SH, Kim TH, Lee HH, Lee A, Jeon DS, Park J, Kim Y. Premenstrual syndrome and premenstrual dysphoric disorder in perimenopausal women. J Menopausal Med. 2014;20:69-74.

3. Brinton RD, Yao J, Yin F, Mack WJ, Cadenas E. Perimenopause as a neurological transition state. Nat Rev Endocrinol. 2015;11:393-405.

4. Ben-Meir A, Yahalomi S, Moshe B, Shufaro Y, Reubinoff B, Saada A. Coenzyme Q-dependent mitochondrial respiratory chain activity in granulosa cells is reduced with aging. Fertil Steril. 2015;104:724-7.

5. Tatone C, Amicarelli E. The aging ovary - the poor granulosa cells. FertilSteril. 2013;99:12-7.

6. Wu YG, Barad DH, Kushnir VA, Lazzaroni E, Wang Q, Albertini DF, Gleicher N Aging-related premature luteinization of granulosa cells is avoided by early oocyte retrieval. J Endocrinol. 2015;226:167-80.

7. van Kasteren YM, Schoemaker J. Premature ovarian failure: a systematic review on therapeutic interventions to restore ovarian function and achieve pregnancy. Hum Reprod Update. 1999;5:483-92.
8. Easley 4th CA, Simerly CR, Schatten G. Stem cell therapeutic possibilities: future therapeutic options for male-factor and female-factor infertility? Reprod Biomed Online. 2013;27:75-80.

9. Danieli P, Malpasso G, Ciuffreda MC, Cervio E, Calvillo L, Copes F, Pisano F, Mura M, Kleijn L, de Boer RA, Viarengo G, Rosti V, Spinillo A, Roccio M, Gnecchi M. Conditioned medium from human amniotic mesenchymal stromal cells limits infarct size and enhances angiogenesis. Stem Cells Transl Med. 2015;4: 448-58.

10. Kim SW, Zhang HZ, Guo L, Kim JM, Kim MH. Amniotic mesenchymal stem cells enhance wound healing in diabetic NOD/SCID mice through high angiogenic and engraftment capabilities. PLoS One. 2012;7:e41105.

11. Volarevic V, Bojic S, Nurkovic J, Volarevic A, Ljujic B, Arsenijevic N, Lako M, Stojkovic M. Stem cells as new agents for the treatment of infertility: current and future perspectives and challenges. Biomed Res Int. 2014; 2014:507234.

12. El-Hayek S, Demeestere I, Clarke HJ. Follicle-stimulating hormone regulates expression and activity of epidermal growth factor receptor in the murine ovarian follicle. Proc Natl Acad Sci U S A. 2014;111:16778-83.

13. Alexander PB, Yuan L, Yang P, Sun T, Chen R, Xiang H, Chen J, Wu H, Radiloff DR, Wang XF. EGF promotes mammalian cell growth by suppressing cellular senescence. Cell Res. 2015;25:135-8.

14. Zamah AM, Hsieh M, Chen J, Vigne JL, Rosen MP, Cedars MI, Conti M. Human oocyte maturation is dependent on LH-stimulated accumulation of the epidermal growth factor-like growth factor, amphiregulin. Hum Reprod. 2010;25:2569-78.

15. Xie L, Tang Q, Yang L, Chen L. Insulin-like growth factor I promotes oocyte maturation through increasing the expression and phosphorylation of epidermal growth factor receptor in the zebrafish ovary. Mol Cell Endocrinol. 2016:419:198-207.

16. Sugimura S, Ritter LJ, Rose RD, Thompson JG, Smitz J, Mottershead DG, Gilchrist RB. Promotion of EGF receptor signaling improves the quality of low developmental competence oocytes. Dev Biol. 2015; 403:139-49.

17. Sirotkin AV. Growth factors controlling ovarian functions. J Cell Physiol. 2011;226:2222-5.

18. Bu R, Uddin S, Bavi P, Hussain AR, Al-Dayel F, Ghourab S, Ahmed M, AlKuraya KS. HGF/C-Met pathway has a prominent role in mediating antiapoptotic signals through AKT in epithelial ovarian carcinoma. Lab Invest. 2011;91:124-37.

19. Enriquez-Cortina C, Almonte-Becerril M, Clavijo-Cornejo D, PalestinoDominquez M, Bello-Monroy O, Nuno N, Lopez A, Buci L, Souza V, Hernandez-Pando R, Munoz L, Gutierrez-Ruiz MC, Gomez-Quiroz LE. Hepatocyte growth factor protects against isoniazid/rifampicin-induced oxidative liver damage. Toxicol Sci. 2013;135:26-36.

20. Ding C, Li H, Wang Y, Wang F, Wu H, Chen R, LV J, Wang W, Huang B. Different therapeutic effects of cells derived from human amniotic membrane on premature ovarian aging depend on distinct cellular biological characteristics. Stem Cell Res Ther. 2017:8:173.

21. Eke G, Mangir N, Hasirci N, MacNeil S, Hasirci V. Development of a UV crosslinked biodegradable hydrogel containing adipose derived stem cells to promote vascularization for skin wounds and tissue engineering. Biomaterials. 2017;129:188-98.

22. Xie C, Jin J, Lv X, Tao J, Wang R, Miao D. Anti-aging effect of transplanted amniotic membrane mesenchymal stem cells in a premature aging model of Bmi-1 Deficiency. Sci Rep. 2015;5:13975.

23. Capobianco V, Caterino M, Laffaldano L, Nardelli C, Sirico A, Del Vecchio L, Martinelli P, Pastore L, Pucci P, Sacchetti L. Proteome analysis of human amniotic mesenchymal stem cells (hA-MSCs) reveals impaired antioxidant ability, cytoskeleton and metabolic functionality in maternal obesity. Sci Rep. 2016;6:25270

24. Li J, Mao Q, He J, She H, Zhang Z, Yin C. Human umbilical cord mesenchymal stem cells improve the reserve function of perimenopausal ovary via a paracrine mechanism. Stem Cell Res Ther. 2017;8:55.

25. Su J, Ding L, Cheng J, Yang J, Li X, Yan G, Sun H, Dai J, Hu Y. Transplantation of adipose-derived stem cells combined with collagen scaffolds restores ovarian function in a rat model of premature ovarian insufficiency. Hum Reprod. 2016;31:1075-86.

26. Uzumcu M, Pan Z, Chu Y, Kuhn PE, Zachow R. Immunolocalization of the hepatocyte growth factor (HGF) system in the rat ovary and the antiapoptotic effect of HGF in rat ovarian granulosa cells in vitro. Reproduction. 2006;132:291-9. 
27. Okada M, Suqita K, Inukai T, Goi K, Kaqami K, Kawasaki K, Nakazzawa S. Hepatocyte growth factor protects small airway epithelial cells from apoptosis induced by tumor necrosis factor-alpha or oxidative stress. Pediatr Res. 2004;56:336-44.

28. Wang $T R$, Yan $L Y$, Yan J, Lu CL, Xia X, Yin TL, Zhu XH, Gao JM, Ding T, Hu WH, Guo HY, Li R, Qiao J. Basic fibroblast growth factor promotes the development of human ovarian early follicles during growth in vitro. Hum Reprod. 2014;29: $568-76$

Submit your next manuscript to BioMed Central and we will help you at every step:

- We accept pre-submission inquiries

- Our selector tool helps you to find the most relevant journal

- We provide round the clock customer support

- Convenient online submission

- Thorough peer review

- Inclusion in PubMed and all major indexing services

- Maximum visibility for your research

Submit your manuscript at www.biomedcentral.com/submit 